\title{
Perceptual reference points for form and orientation in young infants: Anchors or magnets?
}

\author{
PAUL C. QUINN \\ Washington \& Jefferson College, Washington, Pennsylvania
}

\begin{abstract}
In some domains, certain stimuli are especially salient and efficiently encoded and are referred to as reference points. One current issue concerns whether reference points are associated with regions of increased or decreased discriminability and function as either perceptual anchors or magnets. In two experiments utilizing the familiarization/novelty-preference procedure, the question of whether 3- to 4-month-old infants' representations of form and orientation information are structured by perceptual reference points and whether such reference points serve as anchors or magnets is examined. In Experiment 1 , infants displayed above-chance discrimination performance for pairs of form stimuli that were equivalently distinct on a physical basis, but only when one member of each pair was a "good" form (i.e., diamond, square, or triangle). In Experiment 2, infants displayed above-chance discrimination performance for pairs of stimuli differing by $7.5^{\prime \prime}$ of orientation, but only when one member of each pair was either horizontal or vertical. The combined results from the two experiments suggest that "simple" gestalts and main axes (i.e., horizontal and vertical) serve as perceptual anchors in young infants' representations of form and orientation information.
\end{abstract}

Much recent research on the origins of visual cognition and its early development has been concerned with the manner in which young infants form perceptual category representations for classes of stimuli (Madole \& Oakes, 1999; Quinn \& Eimas, 1997). For example, 3- and 4-month-old infants have been shown to form category representations for vertically versus diagonally oriented stimuli (Bomba, 1984; Bornstein, 1982: Quinn \& Bomba, 1986), hues (Bornstein, 1985), classes of geometric shapes (Bomba \& Siqueland, 1983; Quinn, 1987; Younger \& Gotlieb, 1988), realistic, pictorial exemplars of various animal kinds at both global and basic levels (Behl-Chadha, 1996; Eimas \& Quinn, 1994; Quinn, Eimas, \& Rosenkrantz, 1993), and spatial relations, such as above, below, and between (Quinn, 1994; Quinn, Cummins, Kase, Martin, \& Weissman, 1996; Quinn, Norris, Pasko, Schmader, \& Mash, 1999). A key issue in research on perceptual category development is how infants come to extract a common structure from multiple exemplars that share one or more attributes and learn that the various members of the category are to be responded to equivalently.

The focus of the research reported here represents a deviation from the current emphasis on equivalence relations among members of a given class and concentrates instead on how certain stimuli within a category "stand out from the rest" and become associated with special prop-

This research was supported by Grant BCS-0096300 from the National Science Foundation. The author thanks Peter Eimas, Elizabeth Spelke, Jeremy Wolfe, and an anonymous reviewer for helpful comments on an earlier draft of the paper and Laurie Yarzab for assistance in conducting the experiments. Correspondence concerning this article should be addressed to P. C. Quinn, Department of Psychology, Washington \& Jefferson College, Washington, PA 15301 (e-mail: pquinn(w) washjeff.edu). erties. Even in the categorization literature, there has been the recognition that some stimuli may serve as reference points-best examples or ideal types that provide internal structure for the graded quality of mental representations of many categories (e.g., Barsalou, 1985; Medin \& Barsalou, 1987; Rosch, 1973; Rosch \& Mervis, 1975; Samuel, 1982). Rosch (1975), for example, reported that focal colors and main axis orientations (i.e., horizontal and vertical) may serve as reference points in their respective domains. A reference point is believed to organize a domain in that other stimuli in the domain are believed to be perceived in terms of the degree to which they resemble the reference point. Some evidence suggests that categories may form around reference points; a new stimulus is assigned to one category or another on the basis of its relative similarity to one or another reference point (e.g., Grieser \& Kuhl, 1989; Rosch, 1973).

Although it is difficult to identify a standard operational definition for a reference point in the literature, at least four empirically verifiable properties of reference points have been identified. First, reference points may possess preferred perceptual properties that cause them to be attended to a greater degree than other members of a domain (Bornstein, 1978; Humphrey \& Humphrey, 1989). Second, reference points may be encoded more efficiently into memory than other members of a domain (Bell \& Handel, 1976; Bornstein, Ferdinandsen, \& Gross, 1981; Clement \& Varnadoe, 1967; Harris, LeTendre, \& Bishop, 1974; Heider, 1972; Humphrey, Humphrey, Muir, \& Dodwell. 1986; Quinn \& Eimas, 1998; Quinn, Siqueland, \& Bomba, 1985). Third, and of significant interest in the present study, reference points contrast with other members of a domain to a greater or a lesser degree than other members do with each other and may function as either 
perceptual anchors or magnets (Acker, Pastore, \& Hall, 1995; Humphrey et al., 1986; Kuhl, 1991). An anchor effect is present when there is increased discriminability and presumed expansion of the psychological space around the reference point, whereas a magnet effect is present when there is decreased discriminability and presumed shrinkage of psychological space around the reference point. Fourth, and consistent with a magnet effect, reference points may display asymmetries in similarity judgment and discrimination tasks (Krumhansl, 1978, 1979; Miller \& Eimas, 1996; Rosch, 1975; Tversky, 1977). Specifically, higher similarity (and lower discriminability) are in evidence when the reference point stimulus is the standard and the nonreference point stimulus is the comparison than when the converse is true. ${ }^{1}$

The present report is concerned with the ontogeny of reference points - in particular, with whether reference points arise because of inherent biases in our perceptual and memory systems that may, in turn, allow for rapid learning of certain classes of information (see Walton \& Bower, 1993). Evidence from phonetic categories indicates an early (i.e., prelinguistic) functional onset for reference points in the domain of speech (Kuhl, 1991; Miller \& Eimas, 1996), but whether this is the case for categories in the domain of vision remains to be determined. The dimensions of form and orientation were chosen for investigation because of evidence that (1) reference points function in these domains for adults (Pomerantz, 1977; Rosch, 1975; Sebrechts \& Garner, 1981) and (2) young infants develop category representations for simple geometric form classes and vertical and oblique orientations (reviewed in Quinn \& Eimas, 1986), thereby allowing one to establish whether reference points serve as magnets or anchors within their respective classes. Demonstrating the existence of reference points for the domains of form and orientation in the period of early infancy would suggest that their special status is unlikely to have been the result of an extended period of perceptual learning. In addition, if early reference points can be demonstrated in the domain of vision, establishing their properties will allow for comparisons with the characteristics of early reference points in the domain of speech. In this way, one can determine whether reference points function similarly or differently across domains.

\section{EXPERIMENT 1}

A number of studies with adults have suggested that "good" gestalts or coherent structural configurations may serve as reference points for form information (Pomerantz, 1977; Sebrechts \& Garner, 1981). These investigations have indicated that memory for sets of elements (e.g., dots) that make up regular, familiar patterns (e.g., diamond, cross) is superior to that for sets of elements that are configured in random, irregular patterns. There is also evidence for asymmetries in similarity judgments of form information (Tversky, 1977). Similarity is higher when a "poor" form is judged in relation to a "good" form than when a "good" form is judged in relation to a "poor" form. Furthermore, Rosch (1973) has shown that category representations are more likely to form around "good" gestalts than around "poor" ones.

Developmental data have revealed that infants form category representations organized around "good" form prototypes at 3-4 months (Bomba \& Siqueland, 1983; Quinn, 1987) but do not form category representations organized around "poor" form prototypes until 7-10 months of age (Younger \& Gotlieb, 1988). In addition, "good" forms have been shown to attract more attention and to be encoded more rapidly than "poor" forms in 4-montholds (Humphrey \& Humphrey, 1989; Humphrey et al., 1986; Younger \& Gotlieb, 1988). Moreover, discrimination performance between pairs of "good" forms has been found to be superior to discrimination performance between pairs of "poor" forms in infants of the same age range (Humphrey et al., 1986), a finding that is consistent with an anchor effect in the psychological space around "good" forms. However, the physical difference between the pairs of "good" forms was not equivalent to that between the pairs of "poor" forms, thus leaving open the question of whether discriminability-related reference point effects will occur when physical distances between pairs of "good" and "poor" forms are kept constant. At issue in the present study is whether a physical distortion of a "good" pattern contrasts more with the "good" pattern than an equivalent physical distortion of a "poor" pattern contrasts with the "poor" pattern.

To determine whether "good" gestalts function as reference points in the processing of form information by young infants, 3- to 4-month-olds were administered discrimination tasks between visual patterns using the familiarization/novelty-preference procedure. Three "good" gestalts, the presumed reference points, were created by arranging 12 dots into three simple geometric configurations-a diamond, a square, and a triangle. Three distortions, one for each "good" gestalt, were created by moving each dot in the "good" gestalt a predetermined distance in a random direction. Three "poor" gestalts and their corresponding distortions were created in the same way, with the exception that the 12 dots making up each "poor" gestalt were arranged randomly. Six stimulus pairs were thus created-three of which were used for the "good" gestalt discrimination tasks, and three of which were used for the "poor" gestalt discrimination tasks. Figure 1 displays an example of a "good" and a "poor" gestalt and their corresponding distortions. In each discrimination task, each infant was familiarized with one member of a stimulus pair and then given a preference test that paired the familiar stimulus with a novel stimulus. A preference for the novel stimulus implies both memory for the familiar stimulus and the ability to discriminate between it and the novel stimulus (Fagan, 1970; Fantz, 1964).

There are four possible performance trends that can be examined to determine whether "good" gestalts function as reference points. First, if "good" gestalts are perceptually salient to infants, one would expect them to re- 
(A)

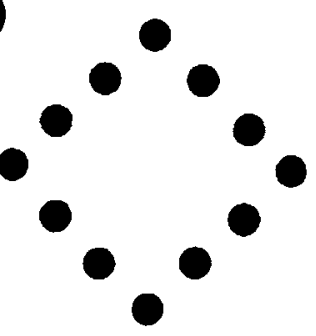

(C)

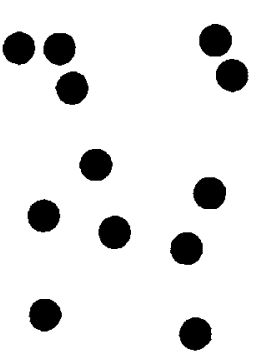

(B)

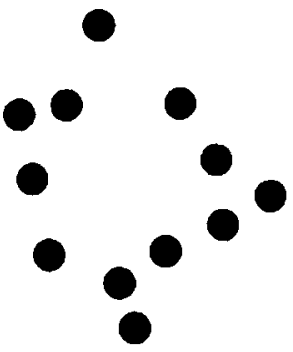

(D)

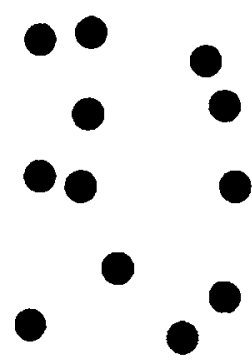

Figure 1. Examples of a "good" form (A) and its distortion (B) and a "poor" form (C) and its distortion (D).

sult in greater initial looking times during the first part of the familiarization phase (Bornstein, 1978; Humphrey \& Humphrey, 1989). Second, on the assumption that "good" gestalts are processed (i.e., encoded) more eff iciently than "poor" gestalts, one would expect that the rate of habituation would be faster for "good" gestalts, relative to "poor" gestalts and to distortions of both "good" and "poor" gestalts (Humphrey et al., 1986). Third, if "good" gestalts function as anchors or magnets in a domain, one would expect overall superior or inferior discrimination performance (i.e., higher or lower novelty preference scores) in the "good" gestalt discrimination task, as compared with the "poor" gestalt discrimination task (Acker et al., 1995; Kuhl, 1991). This expectation is based on the fact that the "good" gestalt will be present in either the familiarization or the preference test phase of the "good" gestalt task, but in neither phase of the "poor" gestalt task. Fourth, if there is a magnet effect, one might also expect asymmetrical discrimination performance (i.e., a short-term magnet effect that is produced during the task and added on to the usual magnet effect; Miller \& Eimas, 1996). Specifically, in the "good" gestalt discrimination task, one would expect inferior discrimination performance (as evidenced by higher novelty preference scores) when the "good" gestalt is the familiar form, relative to when the distortion is familiar (Miller \& Eimas, 1996). However, one would not expect the familiar form (gestalt vs. distortion) to have an effect on discrimination performance in the "poor" gestalt discrimination task, because both the "poor" gestalts and their distortions form arbitrary configurations.

In summary, a comparison of initial looking times, habituation rates, and novelty preference scores for the "good" and "poor" gestalt discrimination tasks should allow one to determine the functional properties of "good" gestalts and whether such gestalts serve as reference points in young infants' representations of visual pattern information.

\section{Method}

Participants. The participants were forty-eight 3- to 4-montholds (mean age $=97$ days, $S D=13$ days), of whom 25 were female and 23 were male. Nine additional infants were tested but failed to complete the procedure because of fussing $(n=8)$ or were excluded from the data analysis because of experimenter error $(n=1)$.

Stimuli. Methods of stimulus preparation followed those described in Quinn (1987). The stimuli consisted of arrangements of 12 black Chartpak dots ( $1.59 \mathrm{~cm}$ in diameter) in known, geometric configurations (a diamond, a square, and a triangle--the "good" gestalts) or in unfamiliar, random configurations (the "poor" gestalts) presented on $17.78 \times 17.78$ white cards cut from Bainbridge $(\# 80)$ posterboard. A single distortion was created for each of the "good" and "poor" gestalts. Distortions were created by moving the points from their original position in the "good" and "poor" gestalts to new positions in the distortions. The magnitude of distortion corresponded to an individual dot displacement of $13.50 \mathrm{~mm}$ (in a random direction) for both "good" and "poor" gestalts. ${ }^{2}$ The mean surface areas (as measured by a planimeter) for the different kinds of patterns were approximately equal and did not differ systematically: $84.67 \mathrm{~cm}(S D=26.56)$ and $80.26 \mathrm{~cm}(S D=17.78)$ for the "good" gestalts and their distortions and $78.20 \mathrm{~cm}(S D=11.17)$ and $86.10 \mathrm{~cm}(S D=15.41)$ for the "poor" gestalts and their distortions (see Figure 1 for examples of the gestaits and their distortions).

Apparatus. The infants were tested with an adaptation of the portable visual preference apparatus described by Fagan (1970). The apparatus consisted of a gray-painted viewing box with a hinged display stage that was used to expose the stimuli to the infant. The display stage contained two laterally separated compartments, into which the stimulus cards could be fitted by an experimenter. The center-to-center distance between the two compartments was $30.50 \mathrm{~cm}$. Midway between the two compartments was a $0.62-\mathrm{cm}$ (diameter) peephole that permitted an experimenter to observe and record the infant's visual fixations on the stimuli. During a trial, when the stage was closed and illuminated by a $60-\mathrm{Hz}$ fluorescent lamp (shielded from the infant's view), the infant could see only the stimuli and the gray background surface of the viewing box.

Procedure. The infants underwent the following general procedure. They were brought to the laboratory by a parent and seated in a reclining position on the parent's lap. There were two experimenters, both of whom were naive with respect to the hypotheses under investigation. The first experimenter positioned the apparatus so that the midline of the infant's head was aligned with the midline of the display stage. At this point with the display stage open, the infant could see the first experimenter from the chest up, in addition to a portion of the room that was painted a light cream color. The first experimenter selected the appropriate stimuli for the forthcoming trial and loaded them into the compartments of the display stage. The first experimenter then elicited the infant's attention and closed the stage, thereby exposing the stimuli to the infant. The center of the stage was approximately $30 \mathrm{~cm}$ above the infant while the stimuli were being viewed.

During the course of a trial, the first experimenter observed the infant through the peephole and recorded visual fixations to the left and right stimuli by means of two 605 XE Accusplit electronic stop 
watches, one of which was held in each hand. Interobserver reliability for Experiments 1 and 2, as determined by comparing the looking times measured by the experimenter using the center peephole and those measured by additional observers using peepholes to the left of the left stimulus compartment and to the right of the right stimulus compartment, averaged .92 .

Between familiarization trials, the first experimenter opened the stage, recorded the data, obtained the infant's attention, centered his or her gaze, and finally closed the stage to begin the next trial. The first and second experimenters changed places for the test trials. The experimenter who presented stimuli and measured the infant's fixations during familiarization now measured trial duration and signaled the end of the test trials, whereas the second experimenter presented the test stimuli and measured the fixations. The two experimenters changed roles across infants. The second experimenter was naive with respect to the familiar stimulus.

The 48 infants were randomly assigned to six experimental conditions with 8 infants in each condition. Three "good" gestalt discrimination conditions were defined by whether one member of the stimulus pair was a "good" gestalt-a diamond, a square, or a triangle. Three "poor" gestalt discrimination conditions were defined by whether both members of a stimulus pair were "poor" gestalts. During the familiarization phase for each condition, each infant was presented with two identical copies of one member of a stimulus pair for six 15-sec trials. During the preference test phase, the novel and familiar members of the stimulus pair were presented simultaneously for two 10 -sec trials. Within each discrimination condition, the member of the stimulus pair that was presented as the familiar stimulus was counterbalanced across infants. Also, within each discrimination condition, the left-right positioning of the novel and familiar stimuli was counterbalanced across infants on the first test trial and reversed on the second test trial.

\section{Results}

Familiarization trials. Individual looking times were summed over both stimuli on each trial and then averaged across the first three trials and the last three trials. The mean looking times are shown in Table 1. An analysis of variance-discrimination condition ("good" vs. "poor") $\times$ familiar stimulus (referent vs. distortion) $\times$ trials (1-3 vs. 4-6)--performed on the individual scores revealed only a main effect of trials $[F(1,44)=29.61$, $p<.001]$. No other effects were significant $[F(1,44)<$ 1.00 , in all cases]. Using the standard operational definition of habituation as a decline in responsiveness with repetitive stimulation (Cohen \& Gelber, 1975), the significant decrement in looking time from the first to the second halves of familiarization indicates that infants in both the "good" and the "poor" gestalt discrimination conditions habituated to the stimuli.

Table 1

Mean Fixation Times (in Seconds) During the Familiarization Trials and Mean Novelty Preference Scores (Percentages) During the Preference Test Trials in Experiment 1

\begin{tabular}{|c|c|c|c|c|c|c|c|}
\hline \multirow{3}{*}{$\begin{array}{l}\text { Discrimination } \\
\text { Condition }\end{array}$} & \multicolumn{4}{|c|}{ Fixation Time } & & & \\
\hline & \multicolumn{2}{|c|}{ Trials $1-3$} & \multicolumn{2}{|c|}{ Trials 4-6 } & \multicolumn{3}{|c|}{ Novelty Preference } \\
\hline & $M$ & $S D$ & $M$ & $S D$ & $M$ & $S D$ & $t^{\mathrm{a}}$ \\
\hline "Good" & 8.03 & 3.39 & 6.19 & 3.47 & 58.72 & 18.48 & $2.31^{*}$ \\
\hline "Poor" & 8.57 & 2.33 & 6.18 & 3.28 & 49.39 & 11.09 & 0.27 \\
\hline
\end{tabular}

Preference test trials. Each infant's looking time to the novel stimulus was divided by the total looking time to both test stimuli and converted to a percentage score by multiplying by 100 . The mean novelty preference scores are shown in Table 1. An analysis of variance-discrimination condition ("good" vs. "poor") $\times$ familiar stimulus (referent vs. distortion)-performed on the individual preference scores yielded only an effect of discrimination condition $[F(1,44)=4.52, p<.05]$, indicating that the mean novelty preference score for the "good" gestalt discrimination condition was reliably higher than that for the "poor" gestalt discrimination condition. Neither the effect of familiar stimulus nor its interaction with discrimination condition was significant $[F(1,44)<2.00, p>.30$, in both cases]. Comparisons of the mean novelty preference scores with the chance value of $50 \%$ indicated that only the infants in the "good" gestalt discrimination condition displayed a mean novelty preference score that was reliably greater than chance.

\section{Discussion}

The pattern of looking times from the familiarization trials did not provide evidence for higher initial looking or more rapid encoding for "good" gestalts, relative to "poor" ones. Equivalent looking times and encoding rates for "good" gestalts and their distortions were likewise observed by Bomba and Siqueland (1983) and Quinn (1987), who presented similar stimuli to 3- to 4-montholds. Younger and Gotlieb (1988) also reported equivalent encoding rates for "good" and "poor" gestalts in 3-montholds but observed higher looking times for "poor" over "good" gestalts in infants of the same age range (although this trend was reversed in 5- to 7-month-olds). Furthermore, Humphrey and Humphrey (1989) observed both higher looking times and more rapid encoding rates for "good" over "poor" gestalts in 4-month-olds. Differences in stimulus metrics used to define "goodness" across the studies (Dodwell \& Caelli, 1985; Garner, 1974; Posner, Goldsmith, \& Welton, 1967) or familiarization procedures (e.g., number and duration of trials) or both may be responsible for some of the inconsistencies in outcomes. A reasonable conclusion regarding the pattern of results from the different studies may be that looking time and encoding rate differences between "good" versus "poor" gestalts are not particularly robust.

An analysis of the mean novelty preference scores from the test trials showed that infants were able to discriminate between "good" gestalts and their distortions, but not between "poor" gestalts and physically equivalent distortions. This finding is consistent with the heightened discriminability around "good" gestalts reported for 4-month-olds by Humphrey et al. (1986) but also extends the previous result by showing that it can be obtained when the physical difference between members of the "good" and the "poor" gestalt discrimination pairings is kept constant. Both sets of outcomes are consistent with "good" forms' serving as perceptual anchors in the 
processing of form information by young infants. ${ }^{3}$ Why reference point stimuli function as magnets in some studies (Kuhl, 1991; Miller \& Eimas, 1996), but as anchors in the present study and others (Acker et al., 1995; Humphrey et al., 1986) will be taken up in the General Discussion section. Also at issue is why, for a given domain, anchor effects tend to be demonstrated for younger participants (i.e., the infants in the present study), whereas older participants display magnet effects (i.e., adults judging the similarity of forms, as described by Tversky, 1977).

\section{EXPERIMENT 2}

Rosch (1975) argued that horizontal and vertical may behave as reference points with magnet or assimilation properties in the processing of orientation information by adults. The evidence for reference points was in this case based on asymmetric similarity relations along the orientation continuum around both the vertical and the horizontal. For example, adult participants judged that a $5^{\circ}$ difference was smaller when the vertical (or the horizontal) was the referent and the deviation was the comparison than when the converse was true. Also relevant here are data from the visual attention literature indicating that adults coarsely code the orientation continuum into vertical (steep), horizontal (shallow), and oblique (tilted) categories during early visual processing (Treisman, 1988, 1993; Treisman \& Gormican, 1988; Wolfe, Friedman-Hill, Stewart, \& O'Connell, 1992). Consistent with these reports, Quinn (1992) observed categoricallike processing of orientation information by adults into vertical, horizontal, and oblique groupings, with boundaries between the classes located in the range from $9^{\circ}$ to $14^{\circ}$ away from the main axes.

There is evidence that the parsing of the orientation continuum into vertical, horizontal, and oblique may begin in early infancy. For example, 3- to 4-month-olds can discriminate between horizontal and vertical, but not between two obliques that are $90^{\circ}$ apart (Quinn et al., 1985). Also, 3- to 4-month-olds have been shown to display a visual pop-out effect for target-distractor contrasts involving vertical and oblique orientations, but not for target-distractor contrasts involving two different oblique orientations (Quinn \& Bhatt, 1998). In addition, Bomba (1984) and Quinn and Bomba (1986) found that 2- to 4-month-olds parse the orientation continuum into a narrow vertical category and a broader oblique category, with the boundary between the two categories lying between $8^{\circ}$ and $11^{\circ}$ away from the vertical. Moreover, Bornstein (1982) reported that 4-month-olds form a category representation for a set of near-vertical orientations extending out to $15^{\circ}$ from either side of the vertical, with the vertical main axis serving as the prototype.

Experiment 2 was modeled after Experiment 1 and was designed to determine whether horizontal and vertical serve as reference points in the processing of orientation information by young infants. Each infant was tested on one of four discrimination tasks involving either the main axis or oblique orientations. In the two main axis tasks, one member of the stimulus pair was either horizontal or vertical, and the other was a $7.5^{\circ}$ deviation. In the two oblique orientation tasks, one member of the stimulus pair was either $45^{\circ}$ or $135^{\circ}$, and the other was a $7.5^{\circ}$ deviation. As in Experiment 1, analyses of looking times during familiarization and novelty preferences during test trials will allow for determination of whether horizontal and vertical display reference point properties in the processing of the orientation continuum by young infants.

\section{Method}

Participants. The participants were sixty-four 3- to 4-month-old infants (mean age $=99$ days, $S D=12$ days), of whom 34 were female and 30 were male. Thirteen additional infants were tested but failed to complete the procedure because of fussing ( $n=11)$ or were excluded from the data analysis because of no comparison between the test stimuli (i.e., $0 \%$ or $100 \%$ preference scores; $n=2$ ).

Stimuli. The stimuli were used previously by Bomba (1984), Quinn and Bomba (1986), and Quinn et al. (1985). They consisted of two square-wave grating (i.e., zebra) patterns made from photographs of black Chartpak stripes on white posterboard. The photographs were cut to fit two circular wooden disks (each $16 \mathrm{~cm}$ in diameter) that could be rotated to place the gratings at the desired orientation. The black and white stripes were $0.80 \mathrm{~cm}$ wide, so that when viewed from a distance of $30 \mathrm{~cm}$, the gratings had a spatial frequency of $0.33 \mathrm{cy} / \mathrm{deg}$, subtended a visual angle of $30.60^{\circ}$, and were near $100 \%$ contrast.

Apparatus. The infants were tested with a visual preference testing apparatus quite similar to the one used in Experiment 1. The two grating disks were mounted onto the compartments on the display stage so that they could be rotated to the desired orientations, using a dial on the back of the stage that indicated the orientation on a protractor. The stage in this case was illuminated by two high-intensity lamps that were shielded from the infant's view.

Procedure. The same general procedure as that used in Experiment 1 was used in Experiment 2. The 64 infants were assigned to four experimental conditions, with 16 infants in each condition. The two main axis discrimination conditions were defined by whether one member of the stimulus pair was either horizontal $\left(0^{\circ}\right)$ or vertical $\left(90^{\circ}\right)$. The two oblique discrimination conditions were defined by whether one member of the stimulus pair was either $45^{\circ}$ or $135^{\circ}$. For all the discrimination conditions, the contrast member of the stimulus pair was a $7.5^{\circ}$ deviation in either direction. Thus, for example, the two deviations for horizontal $\left(0^{\circ}\right)$ were $352.5^{\circ}$ and $7.5^{\circ}$; for vertical $\left(90^{\circ}\right)$, they were $82.5^{\circ}$ and $97.5^{\circ}$; for $45^{\circ}$, they were $37.5^{\circ}$ and $52.5^{\circ}$; and for $135^{\circ}$, they were $127.5^{\circ}$ and $142.5^{\circ}$. During the familiarization phase for each condition, the infants were presented with two identical copies of one member of a stimulus pair for six 15-sec trials. Within each condition, which member of the stimulus pair (i.e., referent vs. deviation) served as the familiar stimulus was

Table 2

Mean Fixation Times (in Seconds) During the Familiarization Trials and Mean Novelty Preference Scores (Percentages) During the Preference Test Trials in Experiment 2

\begin{tabular}{|c|c|c|c|c|c|c|c|}
\hline \multirow{3}{*}{$\begin{array}{l}\text { Discrimination } \\
\text { Condition }\end{array}$} & \multicolumn{4}{|c|}{ Fixation Time } & & & \\
\hline & \multicolumn{2}{|c|}{ Trials $1-3$} & \multicolumn{2}{|c|}{ Trials 4-6 } & \multicolumn{3}{|c|}{ Novelty Preference } \\
\hline & $M$ & $S D$ & $M$ & $S D$ & $M$ & $S D$ & $t \mathrm{a}$ \\
\hline Main axis & 6.78 & 2.94 & 4.48 & 2.45 & 63.96 & 14.47 & $5.46^{*}$ \\
\hline Oblique & 6.05 & 2.70 & 3.94 & 2.32 & 49.03 & 20.12 & -0.27 \\
\hline
\end{tabular}

Note $-t$ a for mean versus chance. ${ }^{*} p<.0005$, one-tailed. 
counterbalanced across infants. During the preference test phase, the novel and the familiar members of the stimulus pair were presented simultaneously for two 10-sec trials. Within each condition, the left-right positioning of the novel and the familiar stimuli was counterbalanced across infants on the first test trial and reversed on the second test trial.

\section{Results}

Familiarization trials. The mean looking times for Trials 1-3 versus 4-6 are shown in Table 2. An analysis of variance-discrimination condition (main axis vs. oblique) $\times$ familiar stimulus (referent vs. deviation) $\times$ trials ( $1-3$ vs. 4-6)-performed on the individual scores revealed only a main effect of trials $[F(1,60)=45.36, p<$ $.001]$. No other effects were significant $[F(1,60)<1.40$, $p>.20$, in all cases]. The significant decrement in looking time from the first to the second halves of familiarization indicates that infants in both the main axis and the oblique discrimination conditions habituated to the stimuli.

Preference test trials. The mean novelty preference scores for the two discrimination conditions are shown in Table 2. An analysis of variance-discrimination condition (main axis vs. oblique) $\times$ familiar stimulus (referent vs. distortion) - performed on the individual preference scores yielded only an effect of discrimination condition $[F(1,60)=11.58, p<.001]$, indicating that the mean novelty preference score for the main axis discrimination condition was reliably higher than that for the oblique discrimination condition. Neither the effect of familiar stimulus nor its interaction with discrimination condition was significant $[F(1,60)<1.8, p>.15$, in both cases]. Only the infants in the main axis discrimination condition displayed a mean novelty preference score that was reliably greater than the chance preference of $50 \%$.

\section{Discussion}

The results of Experiment 2 paralleled those of Experiment 1 . During familiarization, the infants did not display heightened initial looking or more rapid encoding for horizontal and vertical orientations, relative to $7.5^{\circ}$ deviations from the main axis or oblique orientations $\left(45^{\circ}\right.$ and $\left.135^{\circ}\right)$ and their $7.5^{\circ}$ deviations. These outcomes are consistent with those obtained with the same stimuli and similarly aged infants (Bomba, 1984; Quinn \& Bomba, 1986; Quinn et al., 1985).

Data from the novelty preference trials established that infants discriminated $7.5^{\circ}$ deviations from horizontal or vertical, but not equivalent deviations from $45^{\circ}$ or $135^{\circ}$. Horizontal and vertical thus appear to function as perceptual anchors for the processing of orientation information by young infants. ${ }^{4.5 .6}$ The findings complement the evidence that young infants may represent a perceptual category of near vertical orientations with the true vertical as the prototype (Bomba, 1984; Bornstein, 1982; Quinn \& Bomba, 1986). It is interesting to note that the main axes serve as anchors for nearby orientations in the discrimination performance of young infants, but as magnets or attractors for adult judgments of similarity (Rosch,
1975). Whether this apparent change in function of the reference points for orientation reflects a change in task, age, or both will be important to determine in subsequent work.

\section{GENERAL DISCUSSION}

Two experiments were conducted to determine whether "good" gestalts and main axis stimuli display reference point properties in the processing of form and orientation information during the period of early infancy. Although looking time and encoding rate differences did not emerge for the various stimuli presented in either experiment, heightened discriminability was observed in the region of the presumed reference points in both experiments. In Experiment 1,3- to 4-month-olds displayed superior discrimination performance for stimulus pairs made up of "good" gestalts and their distortions, relative to stimulus pairs consisting of "poor" gestalts and physically equivalent distortions. In Experiment 2 , infants from the same age range discriminated $7.5^{\circ}$ changes in the orientation of square-wave grating stimuli, but only when the vertical or the horizontal was presented as the familiar or the novel member of the stimulus pair. The combined findings indicate that "good" gestalts and main axis stimuli function as perceptual anchors in the processing of form and orientation information by young infants. ${ }^{7}$

The results provide additional support for the observation that there may be at least two distinct kinds of reference points, with contrasting discriminability properties (Medin \& Barsalou, 1987). Whereas some reference points behave as anchors that provide separation with neighboring stimuli (e.g., Acker et al., 1995), others act as magnets that attract nearby entities (Kuhl, 1991; Rosch, 1975; Tversky, 1977). When the present demonstration of anchor-type reference points in the domains of visual form and orientation are considered in conjunction with previous reports of magnet-type reference points in young infants in the domain of speech (Kuhl, 1991; Miller \& Eimas, 1996), both sets of findings help to establish that the two kinds of reference points can be observed in early infancy and may be endemic to basic perceptual-cognitive processing operations.

What remains to be determined is why different reference points show different kinds of discriminability properties with respect to neighboring stimuli. One might suggest on the basis of infant studies that anchor effects are more likely to occur in the domain of vision (but see Quinn \& Eimas, 1998), whereas magnet effects are more probable in the domain of speech. However, this possibility does not seem likely, because a broader view of the evidence obtained with adults indicates that magnetlike effects can occur with visual stimuli (Rosch, 1975; Tversky, 1977) and anchorlike effects can occur with speech stimuli (Pastore, 1987). Given that the two kinds of reference points do not appear to be split along domain lines, one might consider the role played by task demands. In this regard, it is interesting to note that in the present ex- 
periments and in one previous demonstration of an anchor effect in the discriminability of musical chords with adults (Acker et al., 1995), participant responses on any given trial were made on the basis of having been presented with both members of a stimulus pair. By contrast, in other studies demonstrating magnetlike effects, participants were responding on any given trial to a single stimulus that would presumably have been related through memory to previous stimuli occurring on earlier trials (Kuhl, 1991; Miller \& Eimas, 1996). This analysis implies that anchor effects may be more likely on discrimination tasks that force more direct perceptual comparisons of the members of a stimulus pair with minimal memory demands, whereas magnet effects may be more commonly observed in discrimination tasks that involve responding to a currently available stimulus on the basis of its similarity to stimuli stored in memory. It leads further to the interesting suggestion that anchor and magnet effects might be observed for the same stimuli in a given age group, depending on task demands (see Tversky, 1977, for a related discussion of how weighting of common vs. distinctive features of pairs of stimuli may change with task context).

One might also observe that within the domains of form and orientation processing, the present studies with young infants provide evidence of perceptual anchor points, whereas previous studies with adults provided evidence for cognitive reference points with magnet-type properties (Rosch, 1973, 1975; Tversky, 1977). It will therefore be important to investigate the age-related determinants of the changeover from anchor- to magnet-type reference points. By way of the analysis offered in the preceding paragraph, it may be that anchor effects are products of early perceptual (bottom-up) processing and a representation based on an initial, first-pass parse of environmental input, whereas magnet effects are outcomes of later cognitive (top-down) processing and a representation based on a more structured abstraction of the input. Although one might object to this suggestion on the grounds that young infants have displayed magnettype effects in the domain of speech (Kuhl, 1991; Miller \& Eimas, 1996), it is possible that these particular magnet effects could have been produced by a greater biological preparedness (and hence, a top-down readiness) for processing the sounds of human language (Eimas \& Miller, 1990) and by experience with sounds from the native language (Kuhl, Williams, Lacerda, Stevens, \& Lindblom, 1992; Polka \& Werker, 1994; see also Quinn \& Eimas, 1998, for further commentary on the role of early experience in the production of magnetlike effects in the visual domain). In contrast, infants in the present experiments would not likely have had prior experience with dot patterns or square-wave gratings.

If anchor effects reflect perceptual operations and magnet effects are more cognitively determined, the following tentative account of the development of anchors and magnets, as well as categories and prototypes, within a domain emerges. Reference points may derive their initial salience because of their psychological separation from other members of the same continuum. In addition, a reference point's distinctiveness may draw attention to a particular region of a physical continuum. Neighboring stimuli are then noticed and initially contrasted, with the reference point thereby giving rise to anchor-type effects. However, perceptual experience with the reference point and nearby stimuli may allow their similarities (e.g., family resemblance) to be represented, thereby giving rise to an internal category structure with a magnetlike reference point (i.e., prototype) at the center. This framework thus relies on perceptual biases (to attend to the distinctive reference point and its immediate surround) and general learning mechanisms (to compute similarity relations around the reference point) to account for the early development of cognitive structure (i.e., category representations).

Whatever account of the anchor and magnet discriminability properties corresponding to various reference points eventually comes to be accepted, the present findings have provided evidence that "good" gestalts and main axis orientations organize the form and orientation continua of young infants by functioning as perceptual anchors in paired-comparison discrimination tasks. Such anchor points may provide the infant with the critical bits of structure necessary to begin the process of building more complex representations of visual input.

\section{REFERENCES}

Acker. B. E., Pastore. R. E.. \& Hal.l. M. D. (1995). Within-category discrimination of musical chords: Perceptual magnet or anchor? Perception \& Psychophysics, 57, 863-874.

BARSAlou, L. W. (1985). Ideals, central tendency, and frequency of instantiation as determinants of graded structure in categories. Journal of Experimental Psychology: Leaming, Memory, \& Cognition, 11 , $629-654$

BeHL-ChADHA. G. (1996). Basic-level and superordinate-like categorical representations in early infancy. Cognition, 60, 105-141.

BEl.1.. H. H.. \& HaNDE.l. S. (1976). The role of pattern goodness in the reproduction of backward masked patterns. Joumal of Experimental Psychology: Human Perception \& Performance, 2, 139-150.

BомвА. P. C. (1984). The development of orientation categories between 2 and 4 months of age. Jounnal of Experimental Child Psychologi: 37, 609-636.

Bumba.P. C. \& Siquel.AND. E. R. (1983). The nature and structure of infant form categories. Jowrnal of Experimental Child Psychology. 35, 294-328.

BoRNSTEIN. M. H. (1978). Visual behavior of the young human infant: Relationships between chromatic and spatial perception and the activity of underlying brain mechanisms. Journal of Experimental Child Psichologr, 26, 174-192.

Bornstrin, M. H. (1982). Perceptual anisotropies in infancy: Ontogenetic origins and implications of inequalities in spatial vision. In H.W. Reese \& L. P. Lipsitt (Eds.), Advances in child development and hehaviof (Vol. 16, pp. 77-123). New York: Academic Press.

Bornsti:IN. M. H. (1985). Infant to adult: Unity to diversity in the development of visual categorization. In J. Mehler \& R. Fox (Eds.), Neonate cognition: Beyond the blooming buzzing confision (pp. 115138), Hillsdale, NJ: Erlbaum.

Bornstein. M. H.. Feridnandsen. K.. \& Gross, C. (1981). Perception of symmetry in infancy. Developmental Psychology, 17, 82-86.

Clement. D. E., \& Varnadoe. K. W. (1967). Pattern uncertainty and the discrimination of visual patterns. Perception \& Psychophysics, 2 , 427-431.

Cohen. L. B. \& Gelbir. E. R. (1975). Infant visual memory. In L. B. 
Cohen \& P. Salapatek (Eds.), Infant perception: From sensation to cognition (Vol. 1, pp. 347-403). New York: Academic Press.

DodWEll, P. C., \& CAElli, T. (1985). Recognition of vector patterns under transformations: Local and global determinants. Quarterly Journal of Experimental Psychology, 37A, 1-23.

Eimas, P. D., \& Miller, J. L. (1990). Infant categories and categorization. In G. M. Edelman, M. Cowan, \& E. Gall (Eds.), Signal and sense: Local and global order in perceptual maps (pp. 433-449). New York: Wiley-Liss.

Eimas, P. D., \& Quinn, P. C. (1994). Studies on the formation of perceptually based basic-level categories in young infants. Child Development, 65, 903-917.

FAGAN, J. F. (1970). Memory in the infant. Journal of Experimental Child Psychology, 9, 217-226.

FANTZ, R. L. (1964). Visual experience in infants: Decreased attention to familiar patterns relative to novel ones. Science, 164, 668-670.

GARNER, W. R. (1974). The processing of information and structure. Potomac, MD: Eribaum.

Grieser, D., \& KUHL, P. K. (1989). Categorization of speech by infants: Support for speech-sound prototypes. Developmental Psychology, 25, 577-588

Harris, P. G., LeTendre, J. B., \& Bishop, A. (1974). The young child's discrimination of obliques. Perception, 3, 261-265.

HEIDER, E. R. (1972). Universals in color naming and memory. Journal of Experimental Psychology, 93, 10-20.

Humphrey, G. K., \& Humphrey, D. E. (1989). The role of structure in infant visual perception. Canadian Journal of Psychology, 43, 165-182.

Humphrey, G. K., Humphrey, D. E., Muir, D., \& Dodwell, P. C. (1986). Pattern perception: Effects of structure and transformation. Journal of Experimental Child Psychology, 41, 128-148.

KRUMHANSL, C. L. (1978). Concerning the applicability of geometric models to similarity data: The interrelationship between similarity and spatial density. Psychological Review, 85, 445-463.

KRUMHANSL, C. L. (1979). The psychological representation of musical pitch in a tonal context. Cognitive Psychology, 11, 346-374.

KuHL, P. K. (1991). Human adults and human infants show a "perceptual magnet effect" for the prototypes of speech categories, monkeys do not. Perception \& Psychophysics, 50, 93-107.

Kuhl, P. K., Williams, K. A., Lacerda, F., Stevens, K. N., \& LindBLOM, B. (1992). Linguistic experience alters phonetic perception in infants by 6 months of age. Science, 255, 606-608.

LeEHEY, S. C., Moskowitz-Cook, A., Brill, S., \& Held, R. (1975). Orientational anisotropy in infant vision. Science, 190, 900-902.

MADOLE, K. L., \& OAKES, L. M. (1999). Making sense of infant categorization: Stable processes and changing representations. Developmental Review, 19, 263-296.

Medin, D. L., \& Barsalou, L. W. (1987). Categorization processes and categorical perception. In S. Harnad (Ed.), Categorical perception: The groundwork of cognition (pp. 455-490). New York: Cambridge University Press.

MilleR, J. L., \& Eimas, P. D. (1996). Internal structure of voicing categories in early infancy. Perception \& Psychophysics, 58, 1157-1167.

Pastore, R. E. (1987). Categorical perception: Some psychophysical models. In S. Harnad (Ed.), Categorical perception: The groundwork of cognition (pp. 29-52). New York: Cambridge University Press.

Polka, L., \& Werker, J. F. (1994). Developmental changes in perception of nonnative vowel contrasts. Journal of Experimental Psychology: Human Perception \& Performance, 20, 42 l-435.

Pomerantz, J. R. (1977). Pattern goodness and speed of encoding. Memory \& Cognition, 5, 235-241.

Posner, M. I., Goldsmith, R., \& Welton, K. E. (1967). Perceived distance and the classification of distorted patterns. Journal of Experimental Psychology, 73, 28-38.

QUiNN, P. C. (1987). The categorical representation of visual pattern information by young infants. Cognition, 27, 145-179.

QuinN, P. C. (1992, November). Orientation perception: Categorical or continuous? Paper presented at the 33rd Annual Meeting of the Psychonomic Society, St. Louis.

QUINN, P. C. (1994). The categorization of above and below spatial relations by young infants. Child Development, 65, 58-69.

QUiNN, P. C., \& BHATT, R. S. (1998). Visual pop-out in young infants:
Convergent evidence and an extension. Infant Behavior \& Development, 21, 273-288.

QuinN, P. C., \& BomBa, P. C. (1986). Evidence for a general category of oblique orientations in four-month-old infants. Journal of Experimental Child Psychology, 42, 345-354.

Quinn, P. C., Cummins. M., Kase, J., Martin, E.. \& Weissman, S. (1996). Development of categorical representations for above and below spatial relations in 3-to 7-month-old infants. Developmental Psychology, 32, 642-650.

QuinN, P. C., \& Eimas, P. D. (1986). On categorization in early infancy. Merrill-Palmer Quarterly, 32, 331-363.

QuinN, P. C., \& Eimas, P. D. (1997). A reexamination of the perceptualto-conceptual shift in mental representations. Review of General Psychology, 1, 271-287.

QUinN, P. C.. \& Eimas. P. D. (1998). Evidence for a global categorical representation of humans by young infants. Journal of Experimental Child Psychology, 69, 151-174.

Quinn, P. C., Eimas, P. D., \& Rosenkrantz, S. L. (1993). Evidence for representations of perceptually similar natural categories by 3 - and 4month-old infants. Perception, 22, 463-475.

Quinn, P. C.. Norris, C. M., Pasko, R. N., Schmader, T. M., \& MASH, C. (1999). Formation of a categorical representation for the spatial relation between by 6- to 7-month-old infants. Visual Cognition, 6. 569-585.

Quinn, P. C., Siqueland, E. R., \& Bomba, P. C. (1985). Delayed recognition memory for orientation by human infants. Journal of Experimental Child Psychology, 40, 293-303.

Rosch, E. H. (1973). Natural categories. Cognitive Psychology, 5, 328350.

Rosch, E. H. (1975). Cognitive reference points. Cognitive Psychologv, $1,532-547$

Rosch, E. H., \& Mervis, C. B. (1975). Family resemblances: Studies in the internal structure of categories. Cognitive Psvchology, 7, 573-605.

Samuel, A. G. (1982). Phonetic prototypes. Perception \& Psychophysics, 31, 307-314.

Sebrechts, M. M., \& Garner, W. R. (1981). Stimulus-specific processing consequences of pattern goodness. Memory \& Cognition, 9, 4l-49.

Treisman, A. (1988). Features and objects: The fourteenth Bartlett Memorial Lecture. Quarterly Journal of Experimental Psychology, 40A, 201-237.

Treisman, A. (1993). The perception of features and objects. In A. Baddeley \& L. Weiskrantz (Eds.), Attention: Selection, awareness, and control (pp. 5-35). Oxford: Oxford University Press, Clarendon Press.

Treisman, A., \& Gormican, S. (1988). Feature analysis in early vision: Evidence from search asymmetries. Psychological Revien, 95, 15-48.

Tversky, A. (1977). Features of similarity. Psychological Revien', 84, 327-352.

Walton, G. E., \& Bower. T. G. R. (1993). Newborns form prototypes in less than one minute. Psychological Scienc'e, 4, 203-205.

Wolfe, J. M., Friedman-Hill, S. R., Stewart, M. l., \& O'Connell., K. M. (1992). The role of categorization in visual search for orientation. Journal of Experimental Psychology: Human Perception \& Performance, 18, 34-49.

YOUNGER, B. A., \& GoTlieb, S. (1988). Development of categorization skills: Changes in the nature or structure of infant form categories? Developmental Psychologv, 24, 611-619.

\section{NOTES}

1. Miller and Eimas (1996) have suggested that asymmetries of this nature may be considered as examples of short-term magnet effects that occur in addition to long-term magnet effects of the sort reported by Kuhl (1991). For example, in the Kuhl task, participants showed inferior discrimination performance between a reference point stimulus and a nonreference point stimulus, relative to two nonreference point stimuli, even though the physical difference between the two pairs of stimuli was equivalent. This poorer discrimination performance was presumed to reflect a permanent shrinkage of the psychological space around the reference point. In contrast, in the Miller and Eimas task, participants displayed inferior discrimination performance when a reference point stimulus was presented as the standard and the nonreference point stim- 
ulus was presented as the comparison than when the converse was true. This effect was interpreted by Miller and Eimas as a temporary alteration of the psychological space around the reference point that provides further shrinkage of that space when the reference point serves as the standard stimulus.

2. For ease of comparison with the language used to describe the stimuli presented to infants in previous work (Quinn, 1987), this dot displacement corresponded to a stimulus referred to in the earlier studies as a Level 30 distortion. One unit of distortion is equivalent to $0.45 \mathrm{~mm}$ of dot displacement, so 30 units of distortion (i.e.. a Level 30 distortion) corresponds with an individual dot displacement of $13.50 \mathrm{~mm}$.

3. The stimulus properties that help to establish a "good" form as a perceptual anchor remain to be determined. In the present case. the "good" form displays appeared to have more complete empty spaces in their middle, a greater degree of closure, and more linear arrangements of elements that might be perceived as lines at low spatial scales. The greater discriminability around these "good" forms could have resulted, at least in part, from detection of these properties.

4. It could be argued that the discrimination results were reflective of a low-level sensory effect-that is, an oblique effect-rather than of a perceptual anchoring effect and that the infants were simply more sensitive to vertical or horizontal gratings, relative to oblique ones. In other words, positive evidence was obtained for discrimination in the main axis-oblique task, but not in the oblique-oblique task, because the main axis gratings were more clearly discernable or of a higher perceived contrast than the obliques. However, if this had been the case, one would have expected visual preferences for the main axis stimuli (Bornstein, 1978: Leehey, Moskowitz-Cook, Brill, \& Held. 1975). Such preferences could have been manifest in the form of higher looking time for the main axes during familiarization trials and a spontaneous preference for the main axes on the test trials. Neither of these outcomes was observed, thus making it unlikely that discrimination performance was simply a matter of sensitivity difference. It should also be noted that the spatial frequency $(0.33 \mathrm{cy} / \mathrm{deg})$ of the grating stimuli was lower than the spatial frequency values where the oblique effect of visual sensitivity has been observed.

5. From a visual attention perspective (e.g., Wolfe et al., 1992), the novelty preferences could be viewed as evidence that the infants noticed the addition of "tilt" in the $\pm 7.5^{\circ}$ stimuli, but did not notice the absence of "tilt" in the $0^{\circ}$ and $90^{\circ}$ stimuli. Around $45^{\circ}$, the $\pm 7.5^{\circ}$ change might not have been enough to allow infants to notice the presence of "steepness" or "shallowness."

6 . It is not clear whether the present data argue for increased discriminability around the reference point or for a special role for the specific reference point. For example, in the case of the orientation continuum, the results of Experiment 2 indicate that a $\pm 7.5^{\circ}$ stimulus can be discriminated from $0^{\circ}$ (i.e., horizontal). However, would a $+3.75^{\circ}$ stimulus be discriminated from a $-3.75^{\circ}$ stimulus (more so than a $41.5^{\circ}$ stimulus would be discriminated from a $48.5^{\circ}$ stimulus)? That is, would one find the high discriminability even if the reference point itself was not one of the stimuli? This is an important question that remains to be answered.

7. One could pose the alternative argument that the "poor" forms from Experiment $I$ and the oblique orientations from Experiment 2 served as "magnets." Although it is difficult to defeat this argument on the basis of data (at least those reported in the present paper), it should be noted that there are many (indeed. an indefinite number of) "poor" forms and a large number of oblique orientations, both of which raise the question: How could they all be magnets? What seems more tractable as an approach is to speak of magnets or anchors with respect to selected stimuli--for example, prototypes, the end locations of continua, and the like - points that can function as locations of attraction or repulsion in psychological space, thus leading to poor or better discrimination when compared with neighboring points.

(Manuscript received August 31, 1999; revision accepted for publication February 16, 2000.) 